\title{
CORRECTIONS
}

\section{What's happening to NHS spending across the UK?}

Figure 1 of this feature article by John Appleby (BMJ

2011;342:d2982, doi:10.1136/bmj.d2982) shows the cut in NHS

Cite this as: $B M J$ 2011;342:d3848

spending planned for Scotland as $-3.3 \%$ whereas in fact it is

$-1.3 \%$ for $2011-2$ 